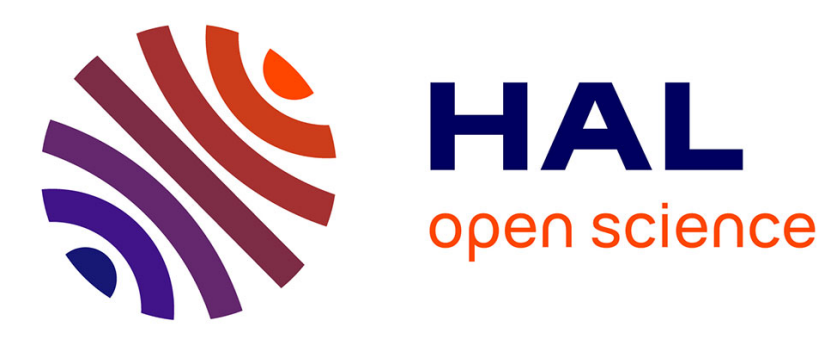

\title{
Interstitial Hydrogen in bcc Binary Alloys: Site Occupancies and Transition Probabilities
}

\author{
A. Biscarini, B. Coluzzi, F. Mazzolai
}

\section{To cite this version:}

A. Biscarini, B. Coluzzi, F. Mazzolai. Interstitial Hydrogen in bcc Binary Alloys: Site Occupancies and Transition Probabilities. Journal de Physique IV Proceedings, 1996, 06 (C8), pp.C8-35-C8-38. 10.1051/jp4:1996805 . jpa-00254522

\section{HAL Id: jpa-00254522 https://hal.science/jpa-00254522}

Submitted on 1 Jan 1996

HAL is a multi-disciplinary open access archive for the deposit and dissemination of scientific research documents, whether they are published or not. The documents may come from teaching and research institutions in France or abroad, or from public or private research centers.
L'archive ouverte pluridisciplinaire HAL, est destinée au dépôt et à la diffusion de documents scientifiques de niveau recherche, publiés ou non, émanant des établissements d'enseignement et de recherche français ou étrangers, des laboratoires publics ou privés. 


\title{
Interstitial Hydrogen in bcc Binary Alloys: Site Occupancies and Transition Probabilities
}

\author{
A. Biscarini, B. Coluzzi and F.M. Mazzolai \\ Department of Physics, University of Perugia, Via A. Pascoli 5, 06100 Perugia, Italy
}

\begin{abstract}
The nature of interstitial sites, their spatial correlations as well as their occupation probabilities by $H$ (or $D$ ) have been investigated in random $b c c$ binary alloys. Sites of both octahedral and tetrahedral types have been taken into account assuming they were distinguishable one another on the basis of either the chemical composition of their first shell of neigbouring atoms or of the distribution of the two metallic species within this shell. Short-range repulsive interactions among $H$ atoms have been accounted for in terms of the hard core model and expressions have been derived for the so-called selective blocking factors. The results of calculations have been used to predict the occurrence of anelastic relaxations due to $H$. A comparison of the theoretical predictions with experimental data available in the literature is presented.
\end{abstract}

\section{INTRODUCTION}

It is well known that the anelastic relaxation provides a powerful spectroscopic technique for investigating the state and motion of point lattice defects [1]. For the case of $H$ there are relaxations occurring in concentrated $b c c$ alloys [2-4], the structural mechanisms of which have not been identified so far. These relaxations have generically been labelled as Snoek relaxations, however, neither the simmetry properties of the dipoles responsible for these relaxations, nor the role played by the two metallic species in determining these simmetry properties have been investigated so far. The main aim of this paper is to develop a statistical model for $\mathrm{H}$ site occupancy and for intersite transitions of $\mathrm{H}$ in random bcc binary alloys starting from previous calculations carried out by Griessen and coworkers [5], and taking into account tetrahedral and octahedral site occupancy, as well as short-range repulsive $\mathrm{H}-\mathrm{H}$ interactions.

\section{DISTRIBUTION OF INTERSTITIAL SITES AND SITE OCCUPANCY}

As shown in Table1, in a random $b c c$ binary alloy $A_{1-y} B_{y}$ can be distinguished twentyfour interstitial sites $I_{u}$, of which eighteen are of octahedral $(O)$ type $(u=1,2, \ldots, 18)$ and six of tetrahedral (T) type $(u=19,20 \ldots, 24)$. The fractions $p_{u}$ of sites of type $u$ are also reported in Table 1 as a function of the alloy composition $y$. The number and nature of the interstitial sites $n$-th nearest neighbours to a given site is indicated in Tables $2 a$ and $2 b$ for distances up to $\sqrt{6} a / 4$ ( $a=$ lattice parameter).

Following the procedure suggested by Brouwer and Griessen [5] the site energies $e_{u}$ for an $H$ atom located on a site of type $u$ (either of octahedral or of tertrahedral type) have been calculated for the $T a_{1-y} N b_{y}, V_{l-y} N b_{y}, T i_{1-y} N b_{y}$ and $T i_{l-y} V_{y}$ alloy systems [6] under the main assumptions that isocompositional atomic clusters embedded in the alloy matrix are energetically degenerate and that all the relevant quantities involved in the calculations vary linearly with composition.

To estimate site populations $c_{u}$, that is, the number of $H$ atoms occupying a given type of site $I_{u}$ divided by the total number $M$ of interstitial sites, the Fermi-Dirac statistics is to be applied to the $\mathrm{H}$ lattice gas [7]. Long-range (attractive) and short-range (repulsive) $H-H$ interactions can be taken into 
account [5] by adding a concentration dependent contribution $f(c)\left(c=\Sigma_{u} c_{u}\right)$ to the site energies $e_{u}$ and by introducing selective blocking factors in order to prevent occupancy of sites too close to an occupied site. The following expressions for $c_{u}$ have recently been derived [8]

$$
\mathrm{c}_{\mathrm{u}}=\frac{\mathrm{p}_{\mathrm{u}}-\mathrm{p}_{\mathrm{u}}^{\mathrm{b}}}{1+\operatorname{mexp}\left\{\left[\mathrm{e}_{\mathrm{u}}+\mathrm{f}(\mathrm{c})-\mu\right] / \mathrm{kT}\right\}} \quad(u=l, 2, \ldots, 24)
$$

where $\mu$ is the chemical potential of $H$ and $p_{u}^{b}$ are the fractions of blocked sites, that is, of sites of type $u$ made unoccupable by $H$ atoms divided by the total number $M$ of sites.

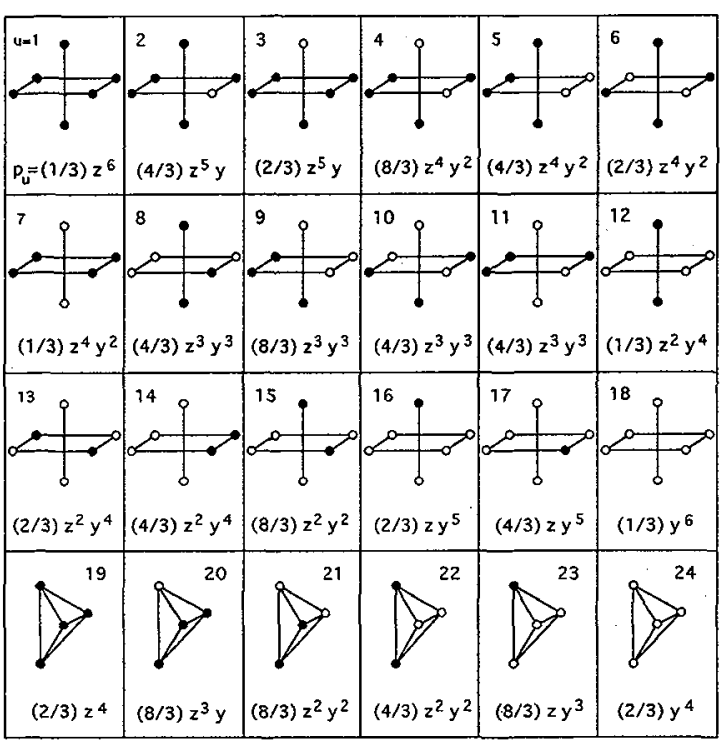

\begin{tabular}{|c|c|c|c|c|}
\hline & $\begin{array}{l}\text { Order of the shell } \\
\text { of neighbours }\end{array}$ & $N^{T n}$ & $\begin{array}{l}\text { Type } \\
\text { of sites }\end{array}$ & d \\
\hline \multirow{5}{*}{ (a) } & first & 2 & 0 & $a / 4$ \\
\hline & second & 4 & $T$ & $\sqrt{2} a / 4$ \\
\hline & third & 2 & $T$ & $a / 2$ \\
\hline & fourth & 4 & 0 & $\sqrt{5} a / 4$ \\
\hline & fitth & 8 & $\mathbf{T}$ & $\sqrt{6} a / 4$ \\
\hline & $\begin{array}{l}\text { Order of the shell } \\
\text { of neighbours }\end{array}$ & $N^{\text {On }}$ & $\begin{array}{l}\text { Type } \\
\text { of sites }\end{array}$ & d \\
\hline \multirow{3}{*}{ (b) } & first & 4 & $T$ & $a / 4$ \\
\hline & second & 4 & 0 & $a / 2$ \\
\hline & third & 8 & $T$ & $\sqrt{5} a / 4$ \\
\hline
\end{tabular}

Table 1: Distinguishable $O$ and $T$ interstitial sites in a binary bcc alloy and associated fractions $p_{u}$

Table 2a: Shells of interstitial sites nearest nieghbour to a given $T$ site, number $\left(\mathrm{N}_{\mathbf{s}}^{\mathrm{T}}\right)$ and type $(\mathrm{T}, \mathrm{O})$ of sites within the shell and distance (d) from the $T$ site.

Table 2b: As in Table $2 a$, but for an $O$ site.

In estimating $p_{u}^{b}$ the assumption will be made that the approaching distance between two $H$ atoms can not be smaller than $0.21 \mathrm{~nm}$ [9]. For the $b c c$ metals $T a, N b$ and $V$ and their alloys the effect is such that an $H$ atom sitting in an $O$ (or $T$ ) site prevents occupancy up to the third (or fifth) shell of $I$ sites. In the limit of the hard core model and for a radius of the core equal to $0.21 \mathrm{~nm}$, suitable approximate expressions for the fractions of blocked sites are the following [8]

$$
\begin{gathered}
p_{u}^{b}=2 Z_{u}^{T 1} \sum_{u^{\prime}=19}^{24} c_{u^{\prime}} q_{u^{\prime} u}^{T 1}+4 Z_{u}^{T 4} \sum_{u^{\prime}=19}^{24} c_{u^{\prime}} q_{u^{\prime} u}^{T 4}+4 Z_{u}^{O 2} \sum_{u^{\prime}=1}^{18} c_{u^{\prime}} q_{u^{\prime} u}^{O 2} \quad(u=1,2, \ldots, 18) \\
p_{u}^{b}=4 Z_{u^{2}}^{T 2} \sum_{u^{\prime}=19}^{24} c_{u^{\prime}} q_{u^{\prime} u}^{T 2}+2 Z_{u}^{T 3} \sum_{u^{\prime}=19}^{24} c_{u^{\prime}} q_{u^{\prime} u}^{T 3}+8 Z_{u}^{T 5} \sum_{u^{\prime}=19}^{24} c_{u^{\prime}} q_{u^{\prime} u}^{T 5}+4 Z_{u}^{O 1} \sum_{u^{\prime}=1}^{18} c_{u^{\prime}} q_{u^{\prime} u}^{O 1}+8 Z_{u}^{O 3} \sum_{u^{\prime}=1}^{18} c_{u^{\prime}} q_{u^{\prime} u}^{O 3} \quad(u=19 \ldots 24)
\end{gathered}
$$

where $q_{u u}^{S n}$, are the conditional probabilities that, given a site of type $u$, another selected site belonging to its $n t h$ shell of neigbouring sites is of type $u^{\prime}$ (index $S_{n}$ stands for $T_{n}$ or $O_{n}$ according to the nature ( $T$ or $O$ ) of site $u$ ). The first term on the right hand side of (2a) represents an approximate estimate of the fraction of $O$ sites of type $u$ blocked by $H$ atoms sitting in $T$ sites, of any kind, and having site $u$ within their $1 s t$ shell of 2 neighbouring sites. Similar meaning is to be attributed, with obvious interchange of indices, to all the other terms in the right hand side of equations (2a) and (2b). The factors $Z^{s i n}$ (blocking factors) account for the fact that more $H$ atoms may partecipate in the blocking process of the same site and represent the weight to be attributed to each atom. Obviuosly, all the $Z^{\text {Sn }}{ }_{u}$ will approach 
unity at infinite $H$ dilution. Expressions for the $q^{S n}{ }_{u u}$, as well as for the complete set of the blocking factors are given elsewhere [6]. As an example, the expression for $Z^{r 2}{ }_{u}$ are reported in the following relations

$$
Z_{u}^{\mathrm{T} 2}=\left[1+\sum_{u^{\prime}=19}^{24} \mathrm{q}_{u u^{\prime}}^{\mathrm{T} 3} \frac{\mathbf{c}_{\mathrm{u}^{\prime}}}{\mathrm{p}_{\mathrm{u}^{\prime}}}+3 \sum_{\mathrm{u}^{\prime}=1}^{18} \mathrm{q}_{\mathrm{uu^{ \prime }}}^{\mathrm{T4}} \frac{\mathrm{c}_{\mathrm{u}^{\prime}}}{\mathrm{p}_{\mathrm{u}^{\prime}}}+6 \sum_{\mathrm{u}^{\prime}=19}^{24} \mathrm{q}_{\mathrm{uu}}^{\mathrm{T} 5} \frac{\mathbf{c}_{\mathrm{u}^{\prime}}}{\mathrm{p}_{\mathrm{u}^{\prime}}}\right]^{-1} \quad(u=19, \ldots, 24)
$$

The sum inside the square brackets represents the average number of atoms participating in the blocking process of a selected blocked site of type $u$, that is, of a site which has at least one $H$ atom within its blocking cloud. This number is calculated adding the contributions of the various shells surrounding the blocked site. In relations (3) the occupation probabilities of the unblocked sites are assumed to be unaffected by the presence of the blocking atom and equal to $c_{u^{\prime}}$.

Relations (1,2 and 3) lead to a non-linear system of 24 equations in the unknown quantities $c_{u}$. This system has been solved numerically by using an iterative procedure in which the 84 parameters $Z_{u}^{\text {sn }}$ were readjusted after each iteration, in such a way to match the values of $c_{u}$ determined in the preceding cycle and the unknown quantity $f(c)-\mu$ was deduced each time from the normalization condition $\Sigma_{\mathrm{u}} \mathrm{c}_{\mathrm{u}}=\mathrm{c}$ The iterative procedure was applied until stable values of $Z_{u}^{s n}$ and $c_{u}$ were obtained. Appropriate initial values for the $Z_{u}^{S n}$ are to be chosen to get convergence of the calculation procedure. To this purpose the system is initially solved for dilute solid solutions of $H(c=0)$, for which all the $Z_{u}^{s n}$ are equal to unity, then, $c$ is incremented step by step up to the desired value, each time initializing $Z_{u}^{s n}$ with the preceding values.

The predictions of the present model and results of Monte Carlo simulations [10], for a simple cubic lattice containing a single atomic species and for blocking effects only extending over the 1st shell of sites showed, are in fairly good agreement up to a fraction of occupable sites (sites neither blocked nor occupied) as low as 0.04 [8].

\section{TRANSITION PROBABILITIES}

The probability for a $H$ direct transition $I_{u} \rightarrow I_{u^{\prime}}$ from a site of type $u$ to one of type $u^{\prime}$, nth $n n$ neighbour of site $u$, is proportional to the joint probability $\pi_{u u^{\prime}}^{S n}$ that, taken a site of type $u$, this is occupied and has an occupable site of type $u^{\prime}$ in its nth shell of neighbouring sites. This joint probability is given by

$$
\pi_{u u^{\prime}}^{S n}=c_{u} q_{u u^{\prime}}^{S n} f_{u u^{\prime}}^{S n}
$$

where $f_{u u^{\prime}}^{S n}$ represents the conditional probability that, given an occupied site $I_{u}$, a site $u^{\prime}$, nth $n n$ of site $u$, is occupable, that is, neither occupied nor blocked. Within the approximation of the blocking model discussed in the previous section, expressions for $f_{u u^{\prime}}^{S n}$ have been derived [8]. As an example the expression of $f_{u u^{\prime}}^{T 2}$ relative to $T$ sites 2 nd $n n$ one of the other ( $\mathrm{T} \rightarrow \mathrm{T}$ transitions) is the following

$$
f_{u u^{\prime}}^{T 2}=1-\left[\sum_{v=19}^{24} q_{u^{\prime} v}^{T 3} \frac{c_{v}}{p_{v}}+3 \sum_{v=1}^{18} q_{u^{\prime} v}^{T 4} \frac{c_{v}}{p_{v}}+6 \sum_{v=19}^{24} q_{u^{\prime} v}^{T S} \frac{c_{v}}{p_{v}}\right] \quad\left(u, u^{\prime}=19, \ldots 24\right)
$$

where the first, second and third sums represent the probabilities that a generic site of the $3 \mathrm{rd}$, 4th and 5 th shell of site $u^{\prime}$ is occupied, respectively. Transitions associated with non-coincident values of $u$ and $u^{\prime}$ lead to changes in the nature of the elastic dipole, thus giving rise to dipole-dipole reactions, those with coincident values of $u$ and $u$ ' cause reorientation of the elastic dipole $I_{u}$. 


\section{ANELASTIC RELAXATIONS}

The height $Q_{M}^{-1}$ of the $H$ internal friction peaks observed in $T i_{75} V_{25}, T i_{50} N b_{50}, V_{50} N b_{50}$ alloy systems at around $100 K[2-4]$ are plotted in Fig. 1 as a function of the $\mathrm{H}$ atomic fraction $n(n=9 c)$. The main features of $Q_{M}^{-1}(n)$ relationships can be accounted for in terms of reorientation of dipoles $\mathrm{I}_{\mathrm{u}}$ $(u=19,20,21,22)$, as can be seen in Fig. 1 , where the lines represent fits obtained assuming for each reorientational internal friction peak a height $Q_{M}^{-1}$ proportional to the corresponding factor $\pi_{u u}^{T 2}$ and using the proportionality constants as fitting parameters. For $T i_{75} V_{25}$ alloy, contributions to the relaxation arising from reorientation of dipoles other than $I_{19}$ (Snoek effect) and $I_{20}$ (substitutional-interstitial reorientation effect) are negligible for $n \leq 0.66$. For the equiatomic alloys $T i_{50} N b_{50}$ and $V_{50} N b_{50}$ contributions from reorientation of dipoles $I_{2 l}$ and $I_{22}$ are to be taken into account for values of $n>0.3$. As a matter of fact, for the $V_{50} N b_{50}$ alloy a good fit to experimental data beyound $n \cong 0.3$ could be obtained including these contributions (full line in Fig. 1), while a fit only considering $I_{I 9}$ and $I_{20}$ dipoles turned out to be fully unadequate (dashed line in Fig. 1). For $T i_{50} N b_{50}$ alloy the solubility limit $n_{\alpha}$ of $H$ in the $\alpha$ phase is smaller than $0.3 \quad\left(n_{\alpha} \cong 0.20\right)$, thus, only $I_{19}$ and $I_{20}$ needed to be considered. The maximum value of $Q^{-1}{ }_{M}(n)$ relationship for Snoek effect (reorientation of dipole $I_{19}$ ) occurs for $n \cong 0.25$ in the $T i_{75} V_{25}$ alloy, as expected from the calculation of $\pi_{1919}^{T 2}$, and is about three times larger than for $T i_{50} N b_{50}$ alloy, which is again in keeping with estimated values of $\pi_{1919}^{T 2}$ factors for these two alloys. These findings support an interpretation attributing the main contribution to anelastic relaxation at low $\mathrm{H}$ contents to a genuine Snoek effect. The fitting curves for the two equiatomic alloys in Fig. 1 roughly overlap one another at low $H$ contents, indicating no substantial difference in the anisotropy factors of $T i$ and $V$ atomic clusters $I_{19}$. The common value of this factor turned out to be approximately equal to 0.08 .

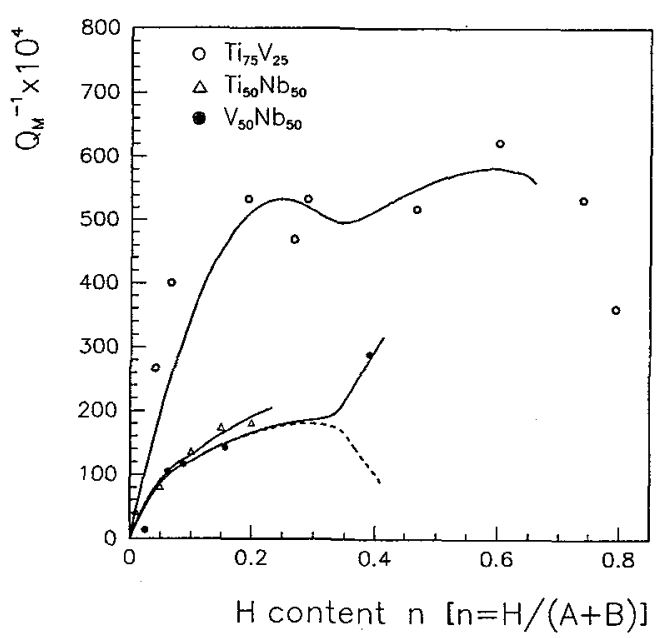

Figure 1: Dependence on $\mathrm{H}$ content $\mathrm{n}$ of the height $\mathrm{Q}^{-1}{ }_{\mathrm{M}}$ of the internal friction peaks observed in the indicated alloy systems. Full lines are fits to the experimental data.

\section{References}

[1] Nowick A.S., Heller W.R., Adv.Phys. 14 (1965) 101

[2] Owen C.V., Buck O., Smith R.P., Peterson D.T., J.de Phys. 46, C10 (1985) 107

[3] Snead C.L., Bethin J., Phys.Rev. B 32 (1985) 4254

[4] Kato K., Tanaka K., Scripta Met. 20 (1986) 1031

[5] Brower R.C.and Griessen R., Phys Rev. B 40 (1989) 1481

[6] Biscarini A., B.Coluzzi and F.M.Mazzolai (to be published)

[7] Kirchheim R., Acta Met. 30 (1982) 1069

[8] Biscarini A., B.Coluzzi and F.M.Mazzolai, Phys.Rev. B 49 (1993) 969

[9] Switendick A.C., Z.Phys.Chem.N.F. 117 (1979) 80

[10] Murch G.E., Phil.Mag. A41 (1980) 701 